\title{
Erratum to: What Is a Procedure?
}

\author{
Eric C.R. Hehner
}

\section{Erratum to:}

Chapter 2 in: M. Mazzara, B. Meyer (eds.), Present and Ulterior Software Engineering, https://doi.org/10.1007/978-3-319-67425-4_2

The original version of chapter 2 was inadvertently published with errors. The presentation of the equations was incorrect. This has been corrected. 